\title{
Generating trees and pattern avoidance in alternating permutations
}

\author{
Joel Brewster Lewis \\ Massachusetts Institute of Technology \\ jblewis@math.mit.edu \\ Submitted: Aug 6, 2011; Accepted: Jan 10, 2012; Published: Jan 16, 2012 \\ Mathematics Subject Classification: 05A05, 05A15
}

\begin{abstract}
We extend earlier work of the same author to enumerate alternating permutations avoiding the permutation pattern 2143. We use a generating tree approach to construct a recursive bijection between the set $A_{2 n}(2143)$ of alternating permutations of length $2 n$ avoiding 2143 and the set of standard Young tableaux of shape $\langle n, n, n\rangle$, and between the set $A_{2 n+1}(2143)$ of alternating permutations of length $2 n+1$ avoiding 2143 and the set of shifted standard Young tableaux of shape $\langle n+2, n+1, n\rangle$. We also give a number of conjectures and open questions on pattern avoidance in alternating permutations and generalizations thereof.
\end{abstract}

\section{Introduction}

The problem of enumerating permutations avoiding a given pattern, first studied systematically by Simion and Schmidt [11], has received a great deal of attention and has led to a number of interesting variations, including the enumeration of special classes of pattern-avoiding permutations. One such variation, first studied by Mansour in [9], is the enumeration of alternating permutations avoiding a given pattern or collection of patterns. Alternating permutations have the intriguing property $[9,15]$ that for any pattern of length three, the number of alternating permutations of a given length avoiding that pattern is a Catalan number.

In $[8,7]$, the present author extended the study of pattern avoidance in alternating permutations to patterns of length four by enumerating the set $A_{2 n}(1234)$ of alternating permutations with no four-term increasing subsequence, and in particular by showing that $A_{2 n}(1234)$ is in bijection with the set of standard Young tableaux of shape $\langle n, n, n\rangle$. One of the two proofs given of this result may be understood as a demonstration that these permutations and tableaux have isomorphic generating trees, that is, that they have the same recursive structure. (The other proof used a modification of the RSK correspondence 
first used by Ouchterlony [10] in the context of doubly-alternating permutations.) In this paper, we show that this approach can be extended to enumerate alternating permutations avoiding the pattern 2143. In particular, we show that $A_{2 n}(2143)$ is in bijection with the set of standard Young tableaux of shape $\langle n, n, n\rangle$ (and so also with $A_{2 n}(1234)$ ) and that $A_{2 n+1}(2143)$ is in bijection with the set of shifted standard Young tableaux of shape $\langle n+2, n+1, n\rangle$. We end with a large number of open problems.

In Section 2, we provide some definitions and notation that will be used throughout the paper. In Section 3, we describe a generating tree for standard Young tableaux of certain shapes and show that it obeys a simple two-parameter labeling scheme. In Section 4.1, we briefly review (without proof) one of the main results of [8], namely that alternating permutations of even length with no four-term increasing subsequence share the same generating tree as the tableaux of Section 3. In Section 4.2, we give the main result of this paper, that alternating permutations of even length avoiding the pattern 2143 also share this generating tree and so are in bijection with alternating permutations of the same length avoiding 1234 and the aforementioned tableaux. In Section 5, we show that similar methods can be used to enumerate alternating permutations of odd length avoiding the pattern 2143, and that the resulting bijection is with shifted Young tableaux of certain shapes. In Section 6 we give several conjectures and open problems relating to pattern avoidance in alternating permutations. Finally, in Section 7 we provide data that forms the basis for several of these conjectures.

An extended abstract of this paper was presented at FPSAC 2010 [6].

\section{Preliminaries}

In this section, we provide important definitions and notation that will be used throughout the rest of the paper.

\subsection{Permutations, partitions, tableaux}

A permutation $w$ of length $n$ is a word containing each of the elements of $[n]=\{1,2, \ldots, n\}$ exactly once. The set of permutations of length $n$ is denoted $S_{n}$. Given a word $w=$ $w_{1} \cdots w_{n}$ and a permutation $p=p_{1} \cdots p_{k} \in S_{k}$, we say that $w$ contains the pattern $p$ if there exists a set of indices $1 \leq i_{1}<i_{2}<\ldots<i_{k} \leq n$ such that the subsequence $w_{i_{1}} w_{i_{2}} \cdots w_{i_{k}}$ of $w$ is order-isomorphic to $p$, i.e., such that $w_{i_{\ell}}<w_{i_{m}}$ if and only if $p_{\ell}<p_{m}$. Otherwise, we say that $w$ avoids $p$. Given a pattern $p$ and a set $S$ of permutations, we denote by $S(p)$ the set of elements of $S$ that avoid $p$. For example, $S_{n}(123)$ is the set of permutations of length $n$ avoiding the pattern 123, i.e., the set of permutations with no three-term increasing subsequence. We denote the size of a set $S$ by $|S|$, so $\left|S_{3}(123)\right|=5$.

A permutation $w=w_{1} w_{2} \cdots w_{n}$ is alternating if $w_{1}<w_{2}>w_{3}<\ldots$. (Note that in the terminology of [13], these "up-down" permutations are reverse alternating while alternating permutations are "down-up" permutations. Luckily, this convention doesn't matter: any pattern result on either set can be translated into a result on the other via complementation, i.e., by considering $w^{c}$ such that $w_{i}^{c}=n+1-w_{i}$. Then results for 
the pattern 132 and up-down permutations are replaced by results for 312 and down-up permutations, and so on.) We denote by $A_{n}$ the set of alternating permutations of length $n$.

A partition is a weakly decreasing, finite sequence of nonnegative integers. We consider two partitions that differ only in the number of trailing zeroes to be the same. We write partitions in sequence notation, as $\left\langle\lambda_{1}, \lambda_{2}, \ldots, \lambda_{n}\right\rangle$.

Given a partition $\lambda=\left\langle\lambda_{1}, \lambda_{2}, \ldots, \lambda_{n}\right\rangle$, the Young diagram of shape $\lambda$ is the left-justified array of $\lambda_{1}+\ldots+\lambda_{n}$ boxes with $\lambda_{1}$ in the first row, $\lambda_{2}$ in the second row, and so on. We identify each partition with its Young diagram and speak of them interchangeably. If $\lambda$ is a Young diagram with $m$ boxes, a standard Young tableau of shape $\lambda$ is a filling of the boxes of $\lambda$ with $[\mathrm{m}]$ so that each element appears in exactly one box and entries increase along rows and columns. We identify boxes in Young diagrams and tableaux using matrix coordinates, so the box in the first row and second column is numbered $(1,2)$. We denote by $\operatorname{SYT}(\lambda)$ the set of standard Young tableaux of shape $\lambda$.

\section{$2.2 \quad$ Generating trees}

Given a sequence $\left\{\Sigma_{n}\right\}_{n \geq 1}$ of nonempty sets with $\left|\Sigma_{1}\right|=1$, a generating tree for this sequence is a rooted, labeled tree such that the vertices at level $n$ are the elements of $\Sigma_{n}$ and the label of each vertex determines the multiset of labels of its children. In other words, a generating tree is one particular type of recursive structure in which heredity is determined by some local data. We are particularly interested in generating trees for which the labels are (much) simpler than the objects they are labeling. In this case, we may easily describe a generating tree by giving the label $L_{1}$ of the root vertex (the element of $\Sigma_{1}$ ) and the succession rule $L \mapsto S$ that gives the set $S$ of labels of the children in terms of the label $L$ of the parent.

Beginning with work of Chung, Graham, Hoggatt, and Kleiman [2], generating trees have been put to good use in the study of pattern-avoiding permutations, notably in the work of West (see, e.g., [16, 17]). The usual approach has been to consider subtrees of the tree of all permutations given by the rule that $v \in S_{n+1}$ is a child of $u \in S_{n}$ exactly when erasing the entry $n+1$ from $v$ leaves the word $u$. Because we are interested here in alternating permutations and the permutation that results from erasing the largest entry of an alternating permutation typically is not alternating, this tree is unsatisfactory. However, the inverse tree, in which we arrive at the children of $u$ by inserting arbitrary values in the last position (rather than inserting the largest value in an arbitrary position), is well-suited to the case of alternating permutations. This motivates the following definitions.

Given a permutation $u \in S_{n}$ and an element $i \in[n+1]$, there is a unique permutation $v=v_{1} v_{2} \cdots v_{n} v_{n+1} \in S_{n+1}$ such that $v_{n+1}=i$ and the word $v_{1} v_{2} \cdots v_{n}$ is order-isomorphic to $u$. We denote this permutation by $u \leftarrow i$ and refer to it as the extension of $u$ by $i$. In other words, the operation of extending $u$ by $i$ replaces each entry $c \geq i$ in $u$ by $c+1$ and then attaches $i$ to the end of the result. For example, $3142 \leftarrow 3=41523$.

Given a pattern $p$ and a permutation $w \in S_{n}(p)$, we say that $c \in[n+1]$ is active or an 
active value for $w$ (with respect to $p$ ) if $w \leftarrow c$ avoids $p$. (This terminology is borrowed from the "usual" case, in which a position is said to be active if one can insert $n+1$ into that position while preserving pattern avoidance.)

There is a natural generating tree structure on alternating permutations of even length analogous to the tree on all permutations mentioned above: given an alternating permutation $u$ of length $2 n$, its children are precisely the alternating permutations $v$ of length $2 n+2$ such that the prefix of $v$ of length $2 n$ is order-isomorphic to $u$. (Of course, there is also a similar tree for alternating permutations of odd length.) Since pattern containment is transitive, the set $\bigcup_{n>1} A_{2 n}(p)$ of alternating permutations of even length avoiding the pattern $p$ is the set of vertices of a subtree. It is these trees (for the patterns 1234 and 2143) that we will consider in Section 4.

\section{A generating tree for tableaux}

For $n \geq 1$, the collection of standard Young tableaux of shape $\langle n, n, n\rangle$ has a simple associated generating tree: given a tableau $S \in \mathrm{SYT}(n, n, n)$, its children are precisely the tableaux $T \in \mathrm{SYT}(n+1, n+1, n+1)$ such that removing the last column of $T$ leaves a tableau that is order-isomorphic to $S$. Notice that the shape of the tree below $S$ is determined entirely by the entries of the last column of $S$, and in particular by the entries $S(1, n)$ and $S(2, n)$ (since $S(3, n)=3 n$ for all $S \in \operatorname{SYT}(n, n, n)$ ). Thus, we wish to choose a labeling for our tree that captures exactly this information. Our choice (one of several reasonable options) is to assign to each $S \in \operatorname{SYT}(n, n, n)$ the label $(a, b)=(3 n+1-S(2, n), 3 n+1-S(1, n))$. This provides a two-label generating tree for

$$
\bigcup_{n \geq 1} \operatorname{SYT}(n, n, n)
$$

whose root (the unique standard Young tableau of shape $\langle 1,1,1\rangle)$ has label $(4-2,4-1)=$ $(2,3)$. In $[8,7]$, the author established (in a more general context) the following result.

Proposition 3.1. The generating tree that we have just described for standard Young tableaux with all columns of length three obeys the rule

$$
(a, b) \mapsto\{(x, y) \mid 2 \leq x \leq a+1 \text { and } x+1 \leq y \leq b+2\}
$$

For example, the root tableau \begin{tabular}{|l|l}
1 & 1 \\
& 2 \\
\hline
\end{tabular} has label $(2,3)$ and has five children,

$$
\begin{array}{|l|l|}
\hline 1 & 4 \\
\hline 2 & 5 \\
\hline 3 & 6 \\
\hline
\end{array}, \begin{array}{|l|l|}
\hline 1 & 3 \\
\hline 2 & 5 \\
\hline 4 & 6 \\
\hline
\end{array}, \begin{array}{|l|l|}
\hline 1 & 2 \\
\hline 3 & 5 \\
\hline 4 & 6 \\
\hline
\end{array}, \begin{array}{|l|l|}
\hline 1 & 3 \\
\hline 2 & 4 \\
\hline 5 & 6 \\
\hline
\end{array} \text { and } \begin{array}{|l|l|}
\hline 1 & 2 \\
\hline 3 & 4 \\
\hline 5 & 6 \\
\hline
\end{array},
$$

which have labels $(2,3),(2,4),(2,5),(3,4)$ and $(3,5)$, respectively. We restate the proof of this result in our current language. 
Proof. Choose a standard Young tableau $S \in \operatorname{SYT}(n, n, n)$ with label $(a, b)$ and a child $T \in \operatorname{SYT}(n+1, n+1, n+1)$ of $S$ with label $(x, y)$. We wish to show that $2 \leq x \leq a+1$ and $x+1 \leq y \leq b+2$. Expressing these conditions in terms of the entries of $S$ and $T$, we must show that

$$
S(2, n)+2 \leq T(2, n+1) \leq 3 n+2
$$

and

$$
S(1, n)+1 \leq T(1, n+1) \leq T(2, n+1)-1 .
$$

We first demonstrate that Equation (1) is valid. Since $T$ is a standard Young tableau, we have $T(2, n+1)<T(3, n+1)=3 n+3$. Thus $T(2, n+1) \leq 3 n+2$, which is the right half of the desired inequality. For the left half, we compute that

$$
\begin{aligned}
T(2, n+1) & =|\{(i, j) \mid T(i, j) \leq T(2, n+1)\}| \\
& \geq \mid\{(i, j) \mid T(i, j) \leq T(2, n) \text { and } j \leq n\} \cup\{(1, n+1),(2, n+1)\} \mid \\
& =|\{(i, j) \mid S(i, j) \leq S(2, n)\} \cup\{(1, n+1),(2, n+1)\}| \\
& =S(2, n)+2,
\end{aligned}
$$

as desired.

Now we demonstrate that Equation (2) is valid. It follows from the relationship between $S$ and $T$ that $S(1, n) \leq T(1, n)$, and since $T$ is a standard Young tableau we have $S(1, n)+1 \leq T(1, n)+1 \leq T(1, n+1) \leq T(2, n+1)-1$, as needed.

We've now established that Equations (1) and (2) hold for all children $T$ of $S$, and so that the labels of the children of $S$ satisfy the claimed inequalities. Now we must show that $S$ has exactly one child with each of these labels.

Given a parent tableau $S$ and prescribed values $T(1, n+1)$ and $T(2, n+1)$ satisfying Equations (1) and (2), we construct the tableau $T$ in the only way possible: define $T(3, n+$ $1)=3 n+3$ and for $1 \leq i \leq 3$ and $1 \leq j \leq n$, set

$$
T(i, j)= \begin{cases}S(i, j), & S(i, j)<T(1, n+1), \\ S(i, j)+1, & T(1, n+1) \leq S(i, j)<T(2, n+1), \\ S(i, j)+2, & T(2, n+1) \leq S(i, j) .\end{cases}
$$

By construction, the object $T$ that results is of shape $\langle n+1, n+1, n+1\rangle$ and contains each of the elements of $[3 n+3]$ exactly once. It is not difficult to check that Equations (1) and (2) imply that $T$ is increasing along rows and columns and consequently that $T \in$ $\operatorname{SYT}(n+1, n+1, n+1)$, and also that $T$ is a child of $S$. Thus, $S$ has at least one child with each label satisfying the given conditions. Finally, it's clear that this $T$ is unique.

\section{Generating trees for $A_{2 n}(1234)$ and $A_{2 n}(2143)$}

Recall from Section 2.2 that for any pattern $p$, there is a natural generating tree for the set $\bigcup_{n} A_{2 n}(p)$ of $p$-avoiding alternating permutations of even length: given a $p$-avoiding 
alternating permutation $u$ of length $2 n$, its children are precisely the $p$-avoiding alternating permutations $v$ of length $2 n+2$ such that the prefix of $v$ of length $2 n$ is order-isomorphic to $u$. In this section, we study this tree for the patterns $p=1234$ and $p=2143$.

\section{$4.1 \quad 1234$}

One of the main results of $[8,7]$ was that even-length alternating permutations with no four-term increasing subsequence share the generating tree for Young tableaux described in Section 3. We now briefly describe this labeling.

Given a 1234-avoiding alternating permutation $w$, assign to it a label $(a, b)$ where $a$ is the smallest entry in $w$ that is the largest entry in a two-term increasing subsequence of $w$, i.e.,

$$
a=\min \left\{w_{j} \mid \exists i<j \text { s.t. } w_{i}<w_{j}\right\},
$$

and $b$ is the number of active values for $w$ with respect to 1234 , i.e., $b$ is the number of choices of $c \in[2 n+1]$ such that $w \leftarrow c$ is 1234-avoiding. (Equivalently, we could define $b$ to be the smallest entry in $w$ that the largest term in a three-term increasing subsequence, or $b=2 n+1$ if $w$ contains no three-term increasing subsequence.) This labeling has previously been used (e.g., by West [16] and Bousquet-Mélou [1]) in the study of $S_{n}(1234)$.

For example, we have for the permutation $w=27583614 \in A_{8}(1234)$ that $\left\{w_{j} \mid \exists i<\right.$ $j$ s.t. $\left.w_{i}<w_{j}\right\}=\{3,4,5,6,7,8\}$ and that the set of active values for $w$ is $\{1,2,3,4\}$. Thus $a=3$ and $b=4$.

As a second example, we have for the permutation $w=68372514 \in A_{8}(1234)$ that $\left\{w_{j} \mid \exists i<j\right.$ s.t. $\left.w_{i}<w_{j}\right\}=\{4,5,7,8\}$ and that $w$ contains no three-term increasing subsequence so all of [9] is active for $w$. Thus $a=4$ and $b=9$.

The root of this tree is the permutation $12 \in A_{2}(1234)$, with label $(2,3)$. Propositions $4.3,4.4$ and 4.6 of [7] collectively establish that if $w \in A_{2 n}(1234)$ has label $(a, b)$ then the collection of labels of children of $w$ is exactly the set of $(x, y)$ such that $2 \leq x \leq a+1$ and $x+1 \leq y \leq b+2$, each pair occurring with multiplicity one.

We now turn our attention to the pattern 2143.

\section{$4.2 \quad 2143$}

In this section, we show that alternating permutations of even length avoiding 2143 have a generating tree isomorphic to those mentioned in preceding sections. A portion of this tree is shown in Figure 1.

Given a permutation $w \in A_{2 n}(2143)$, assign to it a label $(a, b)$ where $a=w_{2 n-1}$ is the next-to-last entry of $w$ and $b$ is the number of active values for $w$ in $[2 n+1]$.

For example, the permutation $w=68142537 \in A_{8}(2143)$ has active values $1,2,3,4,8$ and 9 because the permutations 792536481, 791536482, 791526483, 791526384, 691425378 and 681425379 avoid 2143 while the permutations 691425387,791425386 and 791426385 contain it. Thus for this permutation we have $a=3$ and $b=6$. 


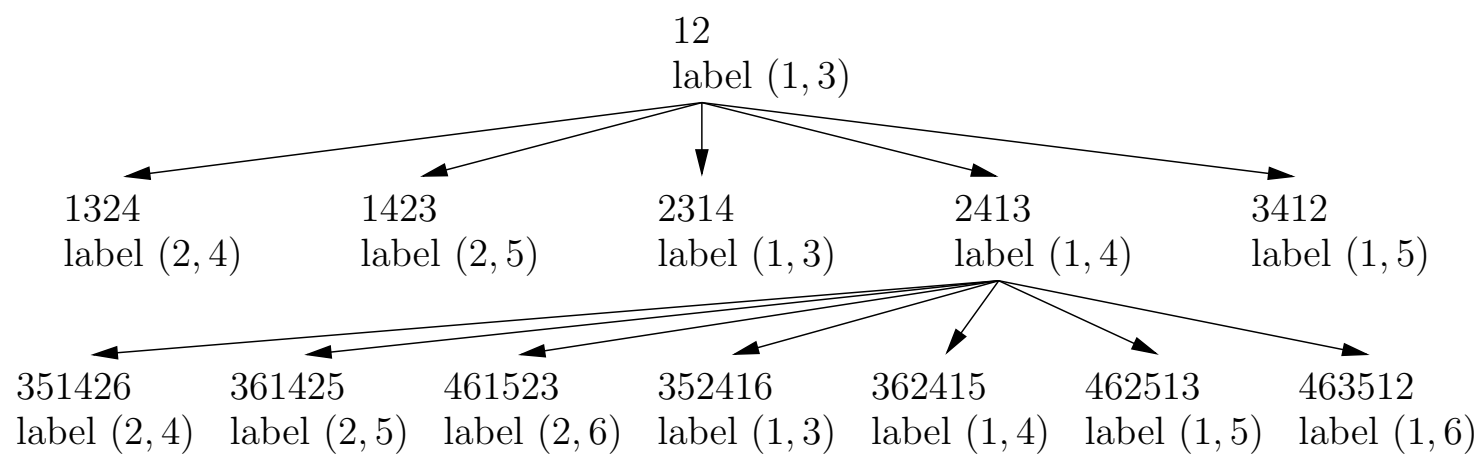

Figure 1: An initial portion of the generating tree for 2143-avoiding alternating permutations of even length.

As a second example, the permutation $w=35462718 \in A_{8}(2143)$ has active values 1 , 2 and 9 . Thus $a=1$ and $b=3$.

We have that the root $12 \in A_{2}(2143)$ has label $(1,3)$. We will show that under this labeling the generating tree for $\bigcup_{n} A_{2 n}(2143)$ obeys the rule

$$
(a, b) \mapsto\{(x, y) \mid 1 \leq x \leq a+1 \text { and } x+2 \leq y \leq b+2\}
$$

and we will use this result to establish an isomorphism between this generating tree and those discussed in the preceding sections. We break the proof of this result into several smaller pieces: Propositions 4.2 and 4.4 form the meat of the argument establishing the succession rule, while Propositions 4.1 and 4.3 are helpful technical lemmas. We begin with a simple observation that will be of use in the subsequent proofs.

Observation. Given a permutation $w \in S_{n}(2143)$, we have that $c \in[n+1]$ is not active for $w$ if and only if there exist $i<j<k$ such that $w_{j}<w_{i}<c \leq w_{k}$.

Proposition 4.1. If $u \in A_{2 n}(2143)$ and $u_{2 n-1}=a$ then $\{1,2, \ldots, a+1\}$ are active values for $u$.

Proof. Fix $u \in A_{2 n}(2143)$ with $u_{2 n-1}=a$, choose $c \leq a+1$ and let $v=u \leftarrow c \in S_{2 n+1}$. We wish to show that $v$ avoids 2143, so suppose otherwise. Then there exist $i<j<k<2 n+1$ such that $v_{i} v_{j} v_{k} c$ is an instance of 2143 in $v$. We use this (suppositional) instance to construct an instance of 2143 in $u$; this contradiction establishes that $c$ is active for $u$. In particular, we show that $u_{i} u_{j} u_{2 n-2} u_{2 n-1}$ is an instance of 2143 in $u$ by showing that $v_{i} v_{j} v_{2 n-2} v_{2 n-1}$ is an instance of 2143 in $v$. In order to do this, it suffices to show that $j<2 n-2$ (so that $v_{i} v_{j} v_{2 n-2} v_{2 n-1}$ is a subsequence of $v$ ) and that $v_{i}<v_{2 n-1}$ (so that this subsequence is order-isomorphic to 2143).

Since $v_{i} v_{j} v_{k} c$ is an instance of 2143 and $c \leq a+1$, we have that $v_{j}<v_{i}<c \leq a+1$ and thus $v_{i} \leq a=u_{2 n-1} \leq v_{2 n-1}$. There are at least three entries to the right of $v_{i}$ in $v$ but only two to the right of $v_{2 n-1}$, so $v_{i} \neq v_{2 n-1}$ and thus $v_{i}<v_{2 n-1}$, one of the two conditions we need. It follows that $v_{j}<v_{i}<v_{2 n-1}<v_{2 n-2}$ and similarly $v_{j}<v_{2 n}$, so $v_{j}$ 
is smaller than all of $v_{2 n-2}, v_{2 n-1}, v_{2 n}$ and $v_{2 n+1}$. These entries form a suffix of $v$, so $v_{j}$ must occur at an earlier position in $v$. That is, we have $j<2 n-2$, the second necessary condition. Thus $v_{i} v_{j} v_{2 n-2} v_{2 n-1}$ is an instance of 2143 in $v$ and so $u_{i} u_{j} u_{2 n-2} u_{2 n-1}$ is an instance of 2143 in $u$. Since $u$ avoids 2143, this is a contradiction, so actually $v$ avoids 2143 and $c$ is active for $u$, as desired.

Proposition 4.2. If $u \in A_{2 n}(2143)$ has label $(a, b)$ and $w$ is a child of $u$ with label $(x, y)$ then $1 \leq x \leq a+1$ and $x+2 \leq y \leq b+2$.

Proof. Suppose that permutations $u \in A_{2 n}$ and $w \in A_{2 n+2}$ have the property that the first $2 n$ entries of $w$ are order-isomorphic to $u$, and set $a=u_{2 n-1}$. We now demonstrate that if $w_{2 n+1}>a+1$ then $w$ contains 2143; this will allow us to conclude the first inequality. Suppose that $w_{2 n+1}>a+1$; then also $w_{2 n}>a+1$ and $w_{2 n+2}>a+1$, while $w_{2 n-1}=a$. Thus $w^{-1}(a+1) \notin\{2 n-1,2 n, 2 n+1,2 n+2\}$ and, defining $i=w^{-1}(a+1)$, we have $i<2 n-1$. Then $w_{i} w_{2 n-1} w_{2 n} w_{2 n+1}$ is an instance of 2143 in $w$. Taking the contrapositive, if $w$ avoids 2143 then $w_{2 n+1} \leq a+1$. Now fix $u \in A_{2 n}(2143)$ with label $(a, b)$ and a child $w \in A_{2 n+2}(2143)$ of $u$ with label $(x, y)$; the preceding argument shows that $x \leq a+1$. Clearly $x \geq 1$, so we have proved the first half of our assertion. We now proceed to bound $y$, the number of active values of $w$.

Define a one-to-one function $f:[2 n] \rightarrow[2 n+2]$ by

$$
f(z)= \begin{cases}z, & z<w_{2 n+1} \\ z+1, & w_{2 n+1} \leq z<w_{2 n+2} \\ z+2, & w_{2 n+2} \leq z\end{cases}
$$

so $f\left(u_{\ell}\right)=w_{\ell}$ for all $\ell \in[2 n]$. We show that $f$ is a map from nonactive values of $u$ to nonactive values of $w$; from this fact we may establish the upper bound on $y$. To this end, choose any $c \in[2 n]$ that is not active for $u$, and choose $i<j<k$ such that $u_{j}<u_{i}<c \leq u_{k}$. We have $\left(w_{i}, w_{j}, w_{k}, f(c)\right)=\left(f\left(u_{i}\right), f\left(u_{j}\right), f\left(u_{k}\right), f(c)\right)$. One can easily see that $f$ preserves order, so $w_{j}<w_{i}<f(c) \leq w_{k}$ and thus $f(c)$ is not active for $w$. Thus for each of the $2 n-b$ choices of a nonactive value $c$ for $u$ we have a corresponding nonactive value $f(c)$ for $w$ and so $w$ has at most $(2 n+2)-(2 n-b)=b+2$ active values, i.e., $y \leq b+2$.

Finally, we have by Proposition 4.1 that $\{1,2, \ldots, x+1\}$ are active values for $w$. We also have that $2 n+3$ is active for $w$ and that $2 n+3 \notin\{1,2, \ldots, x+1\}$, so there are at least $x+2$ active values for $w$. Thus $x+2 \leq y$, which completes the proof of our claim.

So far, we have shown that the only possible labels for a child of a 2143-avoiding permutation are those claimed. The next result shows how the active values of an extension of an alternating 2143-avoiding permutation relate to those of the permutation itself.

Proposition 4.3. Suppose $u \in A_{2 n}(2143)$ has label $(a, b)$ and active values $s_{1}<s_{2}<$ $\ldots<s_{b}$. If $x \leq a+1$ and $v=u \leftarrow x$ then $v \in A_{2 n+1}(2143)$ and $v$ has active values $1, s_{1}+1, s_{2}+1, \ldots, s_{b}+1$. 


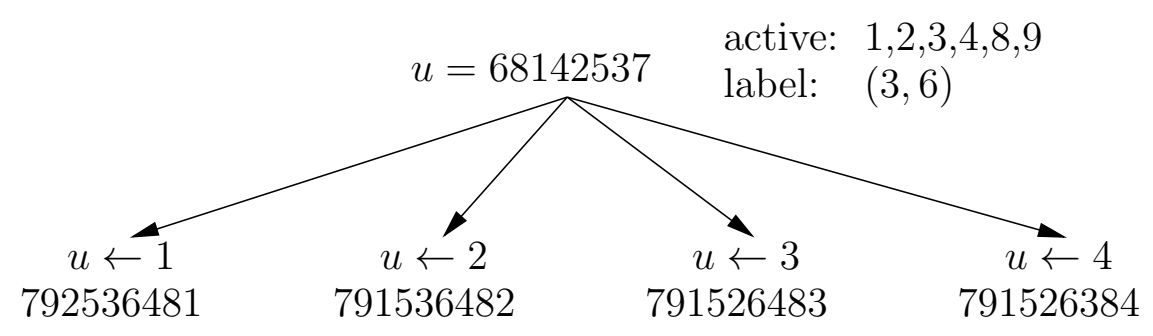

Figure 2: The permutation $68142537 \in A_{8}(2143)$ may be extended by $1,2,3$ or 4 to give an alternating, 2143-avoiding permutation of length 9. Each of the four extensions have active values $1,2,3,4,5,9$ and 10 .

For example, $u=68142537 \in A_{8}(2143)$ has label $(3,6)$ and active values $1,2,3,4,8$ and 9 . For $x=4 \leq 3+1$ we have $v=791526384$ with active values $1,2,3,4,5,9$ and 10. Figure 2 shows the other possible extensions of $u$.

Proof. Choose $u \in A_{2 n}(1234)$ with label $(a, b)$ and choose $x \leq a+1$. Let $s_{1}<\ldots<s_{b}$ be the active values for $u$ and let $v=u \leftarrow x$. Since $v_{2 n+1}=x \leq a+1 \leq v_{2 n-1}+1$ and $v_{2 n-1}<v_{2 n}$, we have $v_{2 n+1} \leq v_{2 n}$ and so actually $v_{2 n+1}<v_{2 n}$. Thus $v$ is alternating. Also, by Proposition 4.1, $x$ is an active value for $u$ and so $v$ avoids 2143. This proves the first part of our claim; to finish, we must show that the set of active values for $v$ is $\left\{1, s_{1}+1, \ldots, s_{b}+1\right\}=\left\{1,2, \ldots, x, s_{x}+1, s_{x+1}+1, \ldots, s_{b}+1\right\}$. We first show that each of these values is in fact an active value for $v$ by considering two cases depending on how the value in question compares to $x$.

Case 1. Fix $m \geq x$ so that $s_{m} \geq x$ is an active value for $u$. We wish to show that $s_{m}+1$ is an active value for $v$. Let $w=w_{1} \cdots w_{2 n+2}$ be the result of extending $v$ by $s_{m}+1$, and suppose for sake of contradiction that $w$ contains an instance $w_{i} w_{j} w_{k} w_{\ell}$ of 2143. We use this suppositional subsequence to find an instance of 2143 in $v$. Since $v$ avoids 2143, we must have $\ell=2 n+2$, so $w_{\ell}=w_{2 n+2}=s_{m}+1$. As $w_{2 n+2}=s_{m}+1>x=v_{2 n+1}$, extending $v$ by $s_{m}+1$ does not change the value of the $(2 n+1)$ th entry and thus $w_{2 n+1}=v_{2 n+1}=x$, whence $w_{2 n+2}>w_{2 n+1}$. Since $w_{k}>w_{2 n+2}$, we have $k \neq 2 n+1$ and so $w_{2 n+1}$ is not part of our instance of 2143 . Let $v^{\prime}$ be the permutation order-isomorphic to $w_{1} w_{2} \cdots w_{2 n} w_{2 n+2}$; we've shown that $v_{i}^{\prime} v_{j}^{\prime} v_{k}^{\prime} v_{2 n+1}^{\prime}$ is an instance of 2143 in $v^{\prime}$. However, we also have that $v^{\prime}=u \leftarrow s_{m}$ and that $s_{m}$ is active for $u$. This is a contradiction, so $w$ cannot contain an instance of 2143 , and we conclude that $s_{m}+1$ is an active value for $v$ by definition.

Case 2. Fix $c \leq x$ so that $c=s_{c}$ is an active value for $u$. We wish to show that $c$ is an active value for $v$. Let $w=w_{1} \cdots w_{2 n+1} w_{2 n+2}$ be the result of extending $v$ by $c$, and suppose for sake of contradiction that $w$ contains an instance $w_{i} w_{j} w_{k} w_{\ell}$ of 2143. Again we use this instance to produce an instance of 2143 in $v$. If $\{k, \ell\} \neq$ $\{2 n+1,2 n+2\}$ then we can conclude by an argument nearly identical to the previous case, so assume that $k=2 n+1$ and $\ell=2 n+2$. Since $w_{i}<w_{k}=w_{2 n+1}$ 


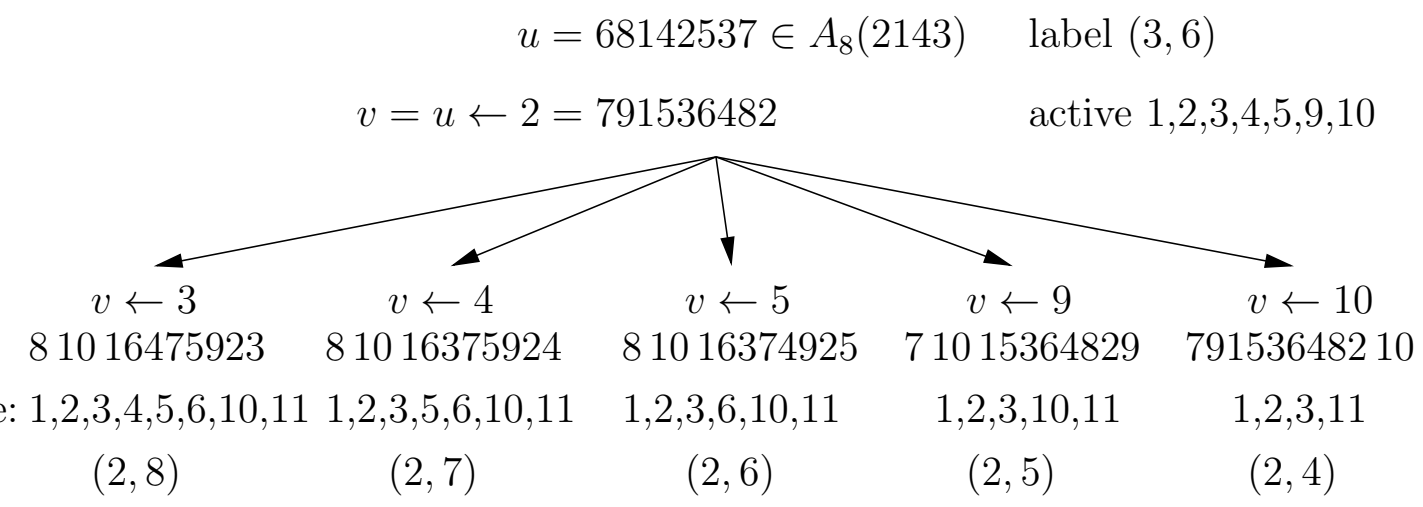

Figure 3: The five children of 68142537 in $A_{10}(2143)$ whose next-to-last entry is 2.

and $w_{j}<w_{k}=w_{2 n+1}$ and $w_{2 n}>w_{2 n+1}$, we have $2 n \notin\{i, j\}$ and so $w_{i} w_{j} w_{2 n} w_{2 n+2}$ is another instance of 2143 in $w$. But now we have an instance not including $w_{2 n+1}$ and so we may proceed as in the previous case. We conclude that $c$ is an active value for $v$.

Finally, we show that these values are the only active values for $v$. In particular, we must show that for every $c>x$ that is not active for $u, c+1$ is not active for $v$. Fix such a $c$. Since $c$ is not active for $u$, there exist $i<j<k$ such that $u_{j}<u_{i}<c \leq u_{k}$. We have $v_{\ell} \leq u_{\ell}+1$ for all $\ell \in[2 n]$, so $v_{j}<v_{i}<c+1$. Since $u_{k}>c-1 \geq x$, we have $v_{k}=u_{k}+1$. Thus $v_{j}<v_{j}<c+1 \leq u_{k}+1=v_{k}$ and so $c+1$ is not an active value for $v$. We conclude that the active values for $v$ are exactly $1,2, \ldots, x, s_{x}+1, s_{x+1}+1, \ldots, s_{b}+1$, as claimed.

Finally, we combine the preceding results to establish that 2143-avoiding alternating permutations of even length have the claimed generating tree.

Proposition 4.4. If $u \in A_{2 n}(2143)$ has label $(a, b)$ and $x, y$ are such that $1 \leq x \leq a+1$, $x+2 \leq y \leq b+2$, then there is a unique child $w \in A_{2 n+2}(2143)$ of $u$ with label $(x, y)$.

Proof. Choose a permutation $u \in A_{2 n}(2143)$ with label $(a, b)$ and choose $(x, y)$ such that $1 \leq x \leq a+1$ and $x+2 \leq y \leq b+2$. We will construct a child of $u$ with label $(x, y)$. This process is partially illustrated in Figure 3 .

Let $s_{1}<\ldots<s_{b}$ be the active values for $u$. Define $v=v_{1} v_{2} \cdots v_{2 n} v_{2 n+1}$ and $w=$ $w_{1} w_{2} \cdots w_{2 n} w_{2 n+1} w_{2 n+2}$ by $v=u \leftarrow x$ and $w=v \leftarrow\left(s_{b+2+x-y}+1\right)$. We claim that $w$ is the desired permutation. We must show that $w$ belongs to $A_{2 n+2}(2143)$ and that its label really is $(x, y)$.

It follows from Proposition 4.3 that $v \in A_{2 n+1}(2143)$. Because $y \leq b+2$, we have $b+2+x-y \geq x$ and so $s_{b+2+x-y}+1>s_{x}=x=v_{2 n+1}$. Thus $w$ is alternating. We also have from Proposition 4.3 that $s_{b+2+x-y}+1$ is an active value for $v$, so $w \in A_{2 n+2}(2143)$. We have left to show that $w$ has label $(x, y)$. 
Since $w_{2 n+2}>v_{2 n+1}$, extending $v$ by $w_{2 n+2}$ leaves the value of the entry in the $(2 n+1)$ th position unchanged and so $w_{2 n+1}=v_{2 n+1}=x$. It remains to show that $w$ has exactly $y$ active values. We claim that the active values for $w$ are precisely $1<2<\ldots<x+1<$ $s_{b+2+x-y}+2<s_{b+3+x-y}+2<\ldots<s_{b}+2$. By Proposition 4.1, we already know that $1,2, \ldots, x+1$ are active for $w$.

For any fixed $i$ such that $b+2+x-y \leq i \leq b$, let $z=w \leftarrow\left(s_{i}+2\right)$. We wish to show that $z$ avoids 2143. We proceed by the same argument as in Case 1 of the proof of Proposition 4.3: since $s_{i}+2>w_{2 n+2}$, we have $z_{2 n+2}=w_{2 n+2}<z_{2 n+3}$. It follows that $z_{2 n+2}$ and $z_{2 n+3}$ cannot be part of the same 2143 pattern in $z$. Thus $z$ contains an instance of 2143 if and only if $z^{\prime}=z_{1} \cdots z_{2 n+1} z_{2 n+3}$ does. However, $z^{\prime}$ is order-isomorphic to $v \leftarrow\left(s_{i}+1\right)$. Since $s_{i}+1$ is an active value for $v, z^{\prime}$ avoids 2143 and so $z$ avoids 2143 . Thus $s_{i}+2$ is an active value for $w$. There are $b-(b+2+x-y)+1=y-x-1$ such active values. We must show that there are no other active values for $w$ larger than $x+1$.

For any fixed $c>s_{b+2+x-y}+2$ that is not of the form $s_{i}+2$, we wish to show that $w \leftarrow c$ contains 2143. We proceed by the same arguments that follow Case 2 of the proof of Proposition 4.3: if $v_{i} v_{j} v_{k}$ are entries of $v$ that can be used to form a 2143 pattern when $v$ is extended by $c-1$ then $w_{i} w_{j} w_{k}$ can be used to form a 2143 pattern when $w$ is extended by $c$.

Finally, for any fixed $c$ such that $x+1<c<s_{b+2+x-y}+2$, let $z=w \leftarrow c$. We wish to show that $z$ contains 2143 . We have that $z_{2 n+1}=x, z_{2 n+2}=s_{b+2+x-y}+2>c$, and $z_{2 n+3}=c>x+1$, and we know that there exists $i<2 n+1$ such that $z_{i}=x+1$. Then $z_{i} z_{2 n+1} z_{2 n+2} z_{2 n+3}=(x+1) x\left(s_{b+2+x-y}+2\right) c$ is an instance of 2143 in $z$, so $c$ is not active for $w$.

The preceding four paragraphs account for all elements $[2 n+3]$. We've shown that exactly $(x+1)+(y-x-1)=y$ of these values are active for $w$. Putting everything together, we have that $w \in A_{2 n+2}(2143)$ is a child of $u$ with $y$ active values and $w_{2 n+1}=x$, i.e., $w$ has label $(x, y)$. In fact, it follows from our proof that every child of $u$ has a distinct label: we've exhausted the possible pairs of values for $w_{2 n+1}, w_{2 n+2}$ such that $w \in A_{2 n+2}(2143)$ is a child of $u$.

Theorem 4.5. For all $n \geq 1$ we have

$$
\left|A_{2 n}(2143)\right|=|\operatorname{SYT}(n, n, n)|=\left|A_{2 n}(1234)\right|=\frac{2 \cdot(3 n) !}{n !(n+1) !(n+2) !} .
$$

Proof. Proposition 3.1 shows that the generating tree for $\bigcup_{n \geq 1} \operatorname{SYT}(n, n, n)$ has root $(2,3)$ and rule

$$
(a, b) \mapsto\{(x, y) \mid 2 \leq x \leq a+1 \text { and } x+1 \leq y \leq b+2\}
$$

while Propositions 4.2 and 4.4 together show that the generating tree for $\bigcup_{n \geq 1} A_{2 n}(2143)$ has root $(1,3)$ and rule

$$
(a, b) \mapsto\{(x, y) \mid 1 \leq x \leq a+1 \text { and } x+2 \leq y \leq b+2\} .
$$

These two trees are isomorphic: replacing each label $(a, b)$ in the first tree with $(a-1, b)$ results in the second tree. Thus, there is a recursive bijection between $A_{2 n}(2143)$ and 
$\operatorname{SYT}(n, n, n)$, and we have the first claimed equality. The work summarized in Section 4.1 gives the second equality. Finally, applying the hook-length formula (see, e.g., [14, Chapter 7.21]) gives the third equality.

\section{$5 \quad$ Generating tree for $A_{2 n+1}(2143)$}

Turning our attention to alternating permutations of odd length, we find that the generating trees of $A_{2 n+1}(1234)$ and $A_{2 n+1}(2143)$ are not isomorphic. Indeed, the two sequences enumerate differently: all sixteen alternating permutations of length five avoid 1234, but only twelve of them avoid 2143. Although this initially seems disappointing, it turns out that we can still use the methods of the preceding section to enumerate 2143-avoiding alternating permutations of odd length.

As in [8], it is convenient to consider the set $A_{2 n+1}^{\prime}$ of down-up alternating permutations of odd length rather than up-down alternating permutations; this switch is permissible because the reverse-complementation map $w=w_{1} \cdots w_{2 n+1} \mapsto\left(2 n+2-w_{2 n+1}\right)(2 n+2-$ $\left.w_{2 n}\right) \cdots\left(2 n+2-w_{1}\right)$ is a bijection between the two sets and preserves 2143-avoidance. Arguments very similar to those of Section 4.2 show that if we associate to the permutation $w \in A_{2 n+1}^{\prime}(2143)$ the label $(a, b)$, where $a=w_{2 n}$ and $b$ is the number of active values for $w$ then the generating tree for $\bigcup_{n \geq 0} A_{2 n+1}^{\prime}(2143)$ has root $1 \in A_{1}^{\prime}(2143)$ with label $(0,2)$ and satisfies the rule

$$
(a, b) \mapsto\{(x, y) \mid 1 \leq x \leq a+1 \text { and } x+2 \leq y \leq b+2\} .
$$

The two children $213,312 \in A_{3}^{\prime}(2143)$ of the root have labels $(1,3)$ and $(1,4)$, respectively, and so on.

We seek to enumerate $A_{2 n+1}^{\prime}(2143)$ by aping our approach for even-length permutations, i.e., by finding a family of objects with isomorphic generating tree that we already know how to enumerate. In the case at hand, these objects turn out to be shifted standard Young tableaux (henceforward SHSYT) of shape $\langle n+2, n+1, n\rangle$. (See [5] or [4, Chapter 10] for definitions, etc.)

Given a SHSYT $T$ of shape $\langle n+2, n+1, n\rangle$, assign to it the label $(a, b)$, where $a=3 n+4-T(2, n+2)$ and $b=3 n+4-T(1, n+2)$. (Note that for $n \geq 1$, we have $T(3, n+2)=3 n+3$ for every SHSYT of this shape, so this label captures all the information we need to reconstruct the last column of $T$.) Then the root of the tree is the unique SHSYT \begin{tabular}{l|l}
\hline & 2 \\
\hline
\end{tabular} of shape $\langle 2,1\rangle$, which has label $(1,2)$. Its children are the two SHSYT

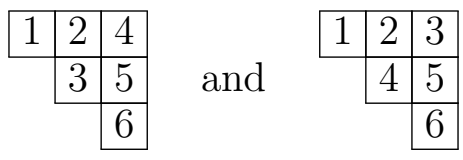

of shape $\langle 3,2,1\rangle$, which have labels $(2,3)$ and $(2,4)$, respectively. In subsequent layers of the tree, the succession rule is identical to the rule for rectangular tableaux; indeed, the subtree below an SHSYT of shape $\langle n+2, n+1, n\rangle$ depends only on its last column (or 
equivalently, on its associated label), and so might as well be the subtree of an SYT of shape $\langle n+1, n+1, n+1\rangle$ with the same label.

It follows immediately that $\left|A_{2 n+1}^{\prime}(2143)\right|$ (and so also $\left|A_{2 n+1}(2143)\right|$ ) is the number of SHSYT of shape $\langle n+2, n+1, n\rangle$. As in the case of standard Young tableaux, there is a simple hook-length formula for SHSYT (see e.g. [5] or [4, pp. 187-190]). In our case, it gives the following result.

Proposition 5.1. For $n \geq 0$ we have

$$
\left|A_{2 n+1}(2143)\right|=\frac{2(3 n+3) !}{n !(n+1) !(n+2) !(2 n+1)(2 n+2)(2 n+3)} .
$$

\section{Open problems}

In this section we pose a number of open enumerative problems related to pattern avoidance in alternating permutations.

\subsection{Generating tree for $A_{2 n}(3412)$}

In this section we lay out a conjectural enumeration for alternating permutations of even length avoiding the pattern 3412. (Alternating permutations of odd length avoiding 3412 are the reversals of alternating permutations of odd length avoiding 2143, so are counted in Section 5.) Given a permutation $w \in A_{2 n}(3412)$, assign to it the label $(a, b)$ where

$$
a=2 n-\max \left\{w_{i} \mid \exists j>i \text { s.t. } w_{i}<w_{j}\right\}
$$

and $b$ is the number of active values (with respect to 3412 ) for $w$ in $[2 n+1]$. We conjecture that this labeling is a the labeling scheme of a generating tree for $\bigcup_{n \geq 1} A_{2 n}(3412)$, as follows.

Conjecture 6.1. If $w \in A_{2 n}(3412)$ is a permutation with label $(a, b)$, then the collection of labels of children of $w$ is exactly the set

$$
\{(x, y) \mid 1 \leq x \leq a+1 \text { and } a+3 \leq y \leq b+2\}
$$

each pair occurring with multiplicity one.

The root of this tree is the permutation $12 \in A_{2}(3412)$ with label $(1,3)$. Its children are the permutations $1324,1423,2314$ and 2413 in $A_{4}(3412)$ with labels $(1,5),(2,5)$, $(1,4)$ and $(2,4)$, respectively.

This conjecture, if proven, leads to a bijection with a family of truncated shifted tableaux and thus to the enumeration of $A_{2 n}(3412)$. We outline the other portions of this argument now. 
For $n \geq 1$, consider the set of tableaux with three rows, $n+1$ boxes in each row, each row shifted one box to the right of the row above it, such that every entry is smaller than the entries below it and to the right. For example,

\begin{tabular}{l|l|l|l|l|}
\hline 1 & 2 & 4 & 6 & \\
\hline & 3 & 5 & 8 & 9 \\
\hline & & 7 & 10 & 11 \\
\cline { 2 - 3 } & & &
\end{tabular}

is such a tableau. The set of these tableaux have a natural generating tree structure in which the tableau $T$ is a child of the tableau $S$ that we get by removing the $(1,1)$, $(2,2)$ and $(3,3)$ entries and applying an order-isomorphism. For example, the tableau of Equation (3) is a child of

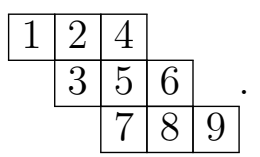

The label of a tableau $T$ is the pair $(T(2,2), T(3,3))$. Thus, the root tableau has label $(3,5)$. The following proposition is easy to verify.

\begin{tabular}{|l|l|l|}
\hline 1 & 2 & \\
\hline & 3 & 4 \\
\hline & 5 & 6 \\
\cline { 2 - 3 }
\end{tabular}

Proposition 6.2. If the tableau $T$ has label $(a, b)$, then the collection of labels of the children of $T$ is exactly the set

$$
\{(x, y) \mid 3 \leq x \leq a+1 \text { and } a+3 \leq y \leq b+2\},
$$

each pair occurring with multiplicity one.

This labeling is not identical to the labeling of Conjecture 6.1, but the two labelings are easy to relate to each other.

Proposition 6.3. The map $(x, y) \mapsto(x+2, y+2)$ is a generating tree isomorphism between the tree given by the labeling of Conjecture 6.1 and the tree of Proposition 6.2. In particular, if Conjecture 6.1 is true then the set $A_{2 n}(3412)$ of alternating permutations of length $2 n$ avoiding the pattern 3412 is in bijection with the set of standard three-row truncated shifted tableaux with $n+1$ boxes in each row.

Finally, by an observation of Greta Panova, these tableaux (and thus conjecturally the permutations in $\left.A_{2 n}(3412)\right)$ are easy to enumerate.

Proposition 6.4 (G. Panova, private communication). The number of standard truncated shifted tableaux with three rows and $n+1$ boxes in each row is

$$
\sum_{i=0}^{n-1} \frac{(2 n+i+1) ! \cdot(n-i+1)(n-i)}{(n+1) ! \cdot n ! \cdot i ! \cdot(2 n+1)(n+i+1)(n+i)} .
$$

Proof. Every standard truncated shifted tableaux with three rows and $n+1$ boxes in each row and $(2, n+2)$-entry equal to $2 n+2+i$ has the following form: 


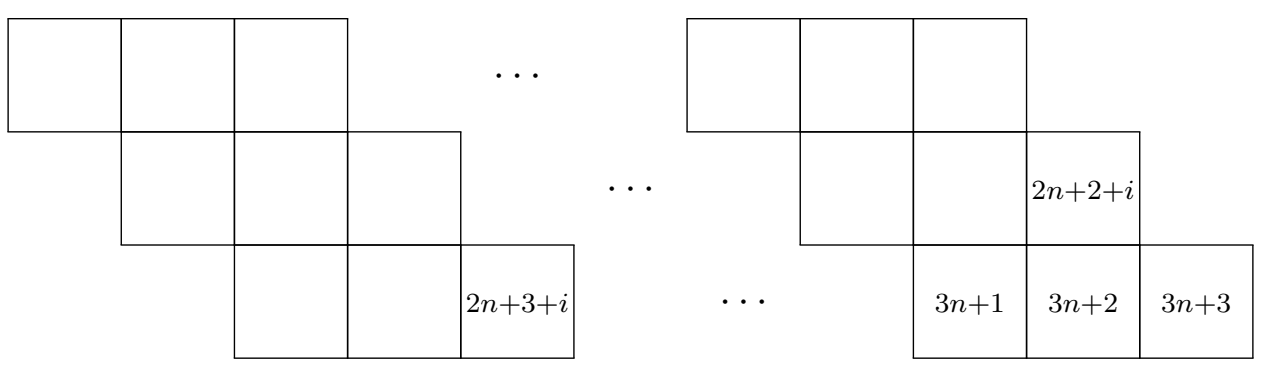

The number of tableaux of this form is precisely the number $g^{\langle n+1, n, i\rangle}$ of SHSYT of shape $\langle n+1, n, i\rangle$. In addition, every standard truncated shifted tableaux $T$ with three rows and $n+1$ boxes in each row satisfies $T(2, n+2)=2 n+2+i$ for some $i \in[0, n-1]$. Thus the total number of such tableaux is

$$
\sum_{i=0}^{n-1} g^{\langle n+1, n, i\rangle}
$$

The desired result follows immediately from the hook-length formula for SHSYT.

\subsection{Other equivalences for patterns of length four}

If permutations $p$ and $q$ satisfy $\left|A_{2 n}(p)\right|=\left|A_{2 n}(q)\right|$ for all $n \geq 1$, we say that $p$ and $q$ are equivalent for even-length alternating permutations. Note that if $p=p_{1} \cdots p_{k}$ and $q=\left(k+1-p_{k}\right)\left(k+1-p_{k-1}\right) \cdots\left(k+1-p_{1}\right)$ (i.e., $p$ and $q$ are reverse-complements) then $p$ and $q$ are equivalent for even-length alternating permutations: for every $n$, the operation of reverse-complementation is a bijection between $A_{2 n}(p)$ and $A_{2 n}(q)$. Pairs of patterns that are equivalent for this reason are said to be trivially equivalent. Similarly, if $\left|A_{2 n+1}(p)\right|=\left|A_{2 n+1}(q)\right|$ for all $n \geq 0$, we say that $p$ and $q$ are equivalent for oddlength alternating permutations, and if $p$ is the reverse of $q$ then we say they are trivially equivalent.

Numerical data (see Section 7) suggest the following conjecture.

Conjecture 6.5. We have $\left|A_{2 n}(p)\right|=\left|A_{2 n}(1234)\right|\left(=\left|A_{2 n}(2143)\right|\right)$ for all $n \geq 1$ and every $p \in\{1243,2134,1432,3214,2341,4123,3421,4312\}$.

Observe that these eight patterns come in four pairs of trivially equivalent patterns. The results of West [16] and computer investigations of short permutations suggest that some of these equivalences may be susceptible to a generating-tree attack. In particular, the generating trees for alternating permutations avoiding 1243 or 2134 may be isomorphic to the generating tree discussed in Sections 3 and 4 .

For alternating permutations of even length, the only other possible equivalences not ruled out by numerical data are captured by the following conjecture.

Conjecture 6.6. We have $\left|A_{2 n}(3142)\right|=\left|A_{2 n}(3241)\right|=\left|A_{2 n}(4132)\right|$ and $\left|A_{2 n}(2413)\right|=$ $\left|A_{2 n}(1423)\right|=\left|A_{2 n}(2314)\right|$ for all $n \geq 1$. 
In both cases, the second of the two equalities is a trivial equivalence. It is interesting to note that the patterns in the first set of equalities are the complements (and also the reverses) of the patterns in the second set of equalities; does this have any significance?

For odd-length alternating permutations, computational data suggest the following conjectures.

Conjecture 6.7. We have $\left|A_{2 n+1}(p)\right|=\left|A_{2 n+1}(1234)\right|$ for all $n \geq 0$ and every $p \in$ $\{2134,4312,3214,4123\}$.

We also have the trivial equivalence $\left|A_{2 n+1}(1234)\right|=\left|A_{2 n+1}(4321)\right|$. The equivalence between 4321 and 4312 may be amenable to generating tree methods.

Conjecture 6.8. We have $\left|A_{2 n+1}(p)\right|=\left|A_{2 n+1}(2143)\right|$ for all $n \geq 0$ and every $p \in$ $\{1243,3421,1432,2341\}$.

We also have the trivial equivalence $\left|A_{2 n+1}(2143)\right|=\left|A_{2 n+1}(3412)\right|$. The equivalence between 3412 and 3421 may be amenable to generating tree methods.

The only other possible equivalence for odd-length alternating permutations not ruled out by data is captured by the following conjecture.

Conjecture 6.9. The permutations 2314,4132,2413,3142, 1423 and 3241 are equivalent for odd-length alternating permutations.

Other than the cases covered by Conjectures 6.5, 6.7 and 6.8, none of the sequences $\left\{\left|A_{2 n}(p)\right|\right\}_{n}$ or $\left\{\left|A_{2 n+1}(p)\right|\right\}_{n}$ for $p \in S_{4}$ are recognizable to the present author (and in particular they do not appear in the OEIS [3]). Numerical data (see Section 7) rule out simple product formulas similar to those of Theorem 4.5 and Proposition 5.1. However, in some cases it may be possible to give a generating tree and so perhaps to adapt the method of [1] to find generating functions or even closed formulas.

\subsection{Other problems}

The conjectures of the preceding section concern two equivalence relations on patterns of length four: equivalence for even-length alternating permutations and equivalence for oddlength alternating permutations. It happens that these relations are both refinements of the usual (Wilf-)equivalence relation for all permutations (see, for example, [12]). (Note that this is true on account of numerical data, regardless of the truth of any of the preceding conjectures.) This suggests the following conjecture.

Conjecture 6.10. If permutations $p$ and $q$ are equivalent for alternating permutations of either parity then $p$ and $q$ are Wilf-equivalent for all permutations.

More broadly, we can ask the following question.

Question 6.11. Are there any large families of patterns that can be shown to be equivalent for alternating permutations (of either parity)? 


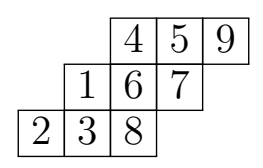

Figure 4: A tableau of "thickened staircase" shape $\langle 5,4,3\rangle /\langle 2,1\rangle$ with reading word $238167459 \in \mathcal{L}_{3,3}$.

The work in [8] suggests two possible generalizations of alternating permutations for purposes of pattern avoidance. One of these (denoted $\mathcal{L}_{n, k}$ in [8]; it consists of the reading words of standard Young tableaux of the "thickened staircase" shape $\langle n+k-1, n+k-$ $2, \ldots, k+1, k\rangle /\langle n-1, n-2, \ldots, 1\rangle$ as in Figure 4) does not seem to give rise to nontrivial equivalences. However, an alternative (and perhaps more natural) generalization is the set Des $_{n, k}$ of permutations of length $n$ with descent set $\{k, 2 k, \ldots\}$, that is, those permutations $w_{1} \cdots w_{n}$ such that $w_{i}>w_{i+1}$ if and only if $i \in\{k, 2 k, \ldots\}$.

Question 6.12. Is $\operatorname{Des}_{n, k}$ a "good" context to study pattern avoidance? In particular, are there any pairs or families of patterns that can be shown to be Wilf-equivalent for these permutations? Is Conjecture 6.10 valid in this context?

In analogy with the case of alternating permutations, it is natural to consider separate cases depending on the congruence class of $n$ modulo $k$. Finally, we note that it may also be fruitful to consider permutations whose descent set is contained in (rather than "is equal to" $\{k, 2 k, \ldots\}$, a case on which our work here does not touch.

\section{Acknowledgements}

We thank Zhao Gu for catching a small error in the proof of Proposition 3.1, and we thank the anonymous referee for several helpful suggestions. 


\section{Appendix}

The tables of data that follow form the basis for several of the conjectures in the preceding section. They were generated by brute-force computer enumeration.

Tables 1 and 2 give the number of alternating permutations avoiding patterns of length four, grouped by conjectural equivalence.

\begin{tabular}{|l||cccccc|}
\hline Patterns & 1 & 3 & 5 & 7 & 9 & 11 \\
\hline \hline$(1234,4321),(2134,4312),(3214,4123)$ & 1 & 2 & 16 & 168 & 2112 & 30030 \\
\hline$(2143,3412),(1243,3421),(1432,2341)$ & 1 & 2 & 12 & 110 & 1274 & 17136 \\
\hline$(2314,4132),(2413,3142),(1423,3241)$ & 1 & 2 & 12 & 106 & 1138 & 13734 \\
\hline$(1324,4231)$ & 1 & 2 & 12 & 110 & 1285 & 17653 \\
\hline$(1342,2431)$ & 1 & 2 & 12 & 108 & 1202 & 15234 \\
\hline$(3124,4213)$ & 1 & 2 & 16 & 168 & 2072 & 28298 \\
\hline
\end{tabular}

Table 1: Values of $A_{n}(p)$ for odd $n$ and $p \in S_{4}$. Parentheses indicate trivial equivalences.

\begin{tabular}{|l||cccccc|}
\hline Patterns & 2 & 4 & 6 & 8 & 10 & 12 \\
\hline \hline $\begin{array}{l}1234,(1243,2134),(1432,3214), \\
2143,(2341,4123),(3421,4312)\end{array}$ & 1 & 5 & 42 & 462 & 6006 & 87516 \\
\hline $3142,(3241,4132)$ & & & & & & \\
\hline$(1423,2314), 2413$ & 1 & 5 & 42 & 444 & 5337 & 69657 \\
\hline 3412 & 1 & 4 & 28 & 260 & 2844 & 34564 \\
\hline 1324 & 1 & 4 & 29 & 290 & 3532 & 49100 \\
\hline$(1342,3124)$ & 1 & 4 & 29 & 292 & 3620 & 51866 \\
\hline$(2431,4213)$ & 1 & 5 & 42 & 453 & 5651 & 77498 \\
\hline 4231 & 1 & 5 & 42 & 454 & 5680 & 78129 \\
\hline 4321 & 1 & 5 & 42 & 462 & 6070 & 90686 \\
\hline & 1 & 5 & 61 & 744 & 10329 & 157586 \\
\hline
\end{tabular}

Table 2: Values of $A_{n}(p)$ for even $n$ and $p \in S_{4}$. Parentheses indicate trivial equivalences. 
Tables 3 and 4 give the number of alternating permutations avoiding certain patterns of length five. Only those patterns that might potentially have nontrivial equivalences are included, and they are grouped by these potential equivalences.

\begin{tabular}{|l||cccccc|}
\hline Patterns & 1 & 3 & 5 & 7 & 9 & 11 \\
\hline \hline$(12534,43521),(21534,43512)$ & 1 & 2 & 16 & 243 & 5291 & 144430 \\
\hline$(12453,35421),(21453,35412)$ & 1 & 2 & 16 & 243 & 5307 & 146013 \\
\hline$(12354,45321),(12543,34521)$, & 1 & 2 & 16 & 243 & 5330 & 148575 \\
$(15432,23451),(21354,45312)$, & & & & & & \\
$(21543,34512),(32154,45123)$ & & & & & & \\
\hline$(12435,53421),(21435,53412)$ & 1 & 2 & 16 & 243 & 5330 & 148764 \\
\hline$(12345,54321),(21345,54312)$, & 1 & 2 & 16 & 272 & 6531 & 194062 \\
$(32145,54123),(43215,51234)$ & & & & & & \\
\hline
\end{tabular}

Table 3: Selected values of $A_{n}(p)$ for odd $n$ and $p \in S_{5}$. Parentheses indicate trivial equivalences. All possible nontrivial equivalences are among the permutations in this table.

\begin{tabular}{|l||cccccc|}
\hline Patterns & 2 & 4 & 6 & 8 & 10 & 12 \\
\hline \hline$(12534,23145),(21534,23154)$ & 1 & 5 & 56 & 997 & 23653 & 679810 \\
\hline$(34512,45123), 45312$ & 1 & 5 & 56 & 1004 & 24310 & 724379 \\
\hline$(12435,13245),(13254,21435)$ & 1 & 5 & 56 & 1004 & 24336 & 727807 \\
\hline$(12453,31245),(21453,31254)$ & 1 & 5 & 61 & 1194 & 30802 & 953088 \\
\hline $12345,21354,(12354,21345)$, & 1 & 5 & 61 & 1194 & 30945 & 970717 \\
$(12543,32145),(15432,43215)$, & & & & & & \\
$(21543,32154),(23451,51234)$, & & & & & & \\
$(34521,54123),(45321,54312)$ & & & & & & \\
\hline
\end{tabular}

Table 4: Selected values of $A_{n}(p)$ for even $n$ and $p \in S_{5}$. Parentheses indicate trivial equivalences. All possible nontrivial equivalences are among the permutations in this table. 
Table 5 gives the number of permutations of length $3 n$ with descent set $\{3,6, \ldots\}$ that avoid certain patterns of length four and five. Only those patterns that might potentially have nontrivial equivalences are included.

\begin{tabular}{|l||cccc|}
\hline Patterns & 3 & 6 & 9 & 12 \\
\hline \hline $2413,(1423,2314)$ & 1 & 9 & 153 & 3465 \\
\hline$(1243,2134),(2341,4123)$ & 1 & 9 & 153 & 3579 \\
\hline $3142,(3241,4132)$ & 1 & 19 & 642 & 27453 \\
\hline $2143,4231,(1432,3214),(3421,4312)$ & 1 & 19 & 642 & 29777 \\
\hline \hline$(12354,21345),(23451,51234)$ & 1 & 19 & 887 & 66816 \\
\hline$(15243,32415),(35241,52413)$ & 1 & 19 & 1077 & 102051 \\
\hline$(12543,32154),(34521,54123)$ & 1 & 19 & 1134 & 114621 \\
\hline 21354,52341$),(21543,32154),(25431,53214)$, & 1 & 19 & 1513 & 211425 \\
\hline$(15432,43215),(215)$, & & & & \\
$(31542,42153),(32541,52143),(35421,54213)$, & & & & \\
$(41532,43152),(42531,53142),(43251,51432)$, & & & & \\
$(43521,54132),(45321,54312),(52431,53241)$, & & & & \\
$(53421,54231)$ & & & & \\
\hline
\end{tabular}

Table 5: Selected values of $\operatorname{Des}_{n, 3}(p)$ for $n$ divisible by 3 and $p \in S_{4}$ or $S_{5}$. Parentheses indicate trivial equivalences. All possible nontrivial equivalences are among the permutations in this table.

\section{References}

[1] M. Bousquet-Mélou. Four classes of pattern-avoiding permutations under one roof: generating trees with two labels. Electronic J. Combinatorics, 9:R19, 2003.

[2] F. R. K. Chung, R. L. Graham, V. E. Hoggatt, Jr., and M. Kleiman. The number of Baxter permutations. J. Combinatorial Theory, Series A, 24:382-394, 1978.

[3] The OEIS Foundation. The on-line encyclopedia of integer sequences. Available online at http://oeis.org/, 2011.

[4] P. N. Hoffman and J. F. Humphreys. Projective representations of the symmetric groups. Oxford University Press, 1992.

[5] C. Krattenthaler. Bijective proofs of the hook formulas for the number of standard Young tableaux, ordinary and shifted. Electronic J. Combinatorics, 2:R13, 1995.

[6] J. B. Lewis. Pattern avoidance in alternating permutations and tableaux (extended abstract). In Proc. 22nd FPSAC, pages 391-402. Discrete Math and Theoretical Computer Science, 2010. 
[7] J. B. Lewis. Pattern avoidance and RSK-like algorithms for alternating permutations and Young tableaux. arXiv:0909.4966v3, 2011.

[8] J. B. Lewis. Pattern avoidance for alternating permutations and Young tableaux. J. Combinatorial Theory, Series A, 118(4):1436-1450, 2011.

[9] T. Mansour. Restricted 132-alternating permutations and Chebyshev polynomials. Annals of Combinatorics, 7:201-227, 2003.

[10] E. Ouchterlony. Pattern avoiding doubly alternating permutations. Proc. FPSAC 2006. arXiv:0908.0255v1.

[11] R. Simion and F. W. Schmidt. Restricted permutations. European J. Combinatorics, 6:383-406, 1985.

[12] Z. Stankova and J. West. A new class of Wilf-equivalent permutations. J. Algebraic Combinatorics, 15:271-290, 2002.

[13] R. P. Stanley. Enumerative Combinatorics, Volume 1. Cambridge University Press, 1997.

[14] R. P. Stanley. Enumerative Combinatorics, Volume 2. Cambridge University Press, 2001.

[15] R. P. Stanley. Catalan addendum to Enumerative Combinatorics. Available online

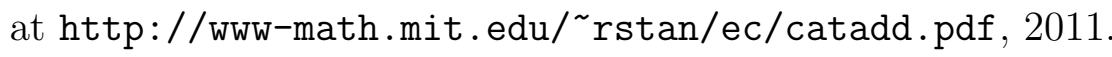

[16] J. West. Generating trees and the Catalan and Schröder numbers. Discrete Mathematics, 146:247-262, 1995.

[17] J. West. Generating trees and forbidden subsequences. Discrete Mathematics, 157:363-372, 1996. 